\title{
ZAYENDA CIVAKî \\ Û ÎMAJA JINA BAŞ-NEBAŞ DI GOTINÊN PÊŞIYAN YÊN KURDAN DE
}

\author{
Tekin Çiftçi \\ Hacı Hamdiye Özdemir Ortaokulu, Artuklu/Mardin, \\ tekincifci@gmail.com•0RCID ID:0000-0002-1003-1711 \\ Article Types / Makale Türü \\ Research Article / Araştırma Makalesi \\ Received / Makale Geliş Tarihi \\ 09/05/2021 \\ Accepted / Kabul Tarihi \\ 29/08/2021
}

https://doi.org/10.26791/sarkiat.935312 


\section{ZAYENDA CIVAKî Û ÎMAJA JINA BAŞ-NEBAŞ DI GOTINÊN PÊŞIYAN YÊN KURDAN DE}

\section{KURTE}

Gotinên pêşiyan, wekî gotinên watedar, qalibgirtî, kurt û bi huner in ku ji cerebe, pêşdaraz û baweriyên batil ên civakan diyar dikin têne penasekirin. Di çanda Kurdan de bi hezaran gotinên pêşiyan hene. Ev gotinên pêşiyan rewşa aborî, çandî, civakî, derûnî, hişmendiya zayenda civakî, weynên zayenda civakî û jiyana rojane ya civakê ji her aliyî ve sererast dikin. Kar û pîşe li gorî weynên zayenda civakî di nav mêr û jinan de hatine dabeşkirin. Wekî her qada jiyanê, di gotinên pêşiyan yên Kurdan de jî weynên zayenda civakî derdikevin pêşiya me. Îmaja jina baş û nebaş li gorî pederşahiyê hatiye şîrovekirin. Ev xebat, di çarçoveya zayenda civakî de li ser îmaja jina "baş" û "nebaş" di gotinên pêşiyan yên Kurdan de hûr dibe. Bi vê mebestê, bi hezaran gotinên pêşiyan yên Kurdan ku di çavkaniyên cuda (nivîskî-devkî) de cih girtine hatine raçavkirin, gotinên pêşiyan ku di çarçoveya zayenda civakî û weynên zayenda civakî de hatine gotin hatine diyarkirin. Di encamê de hate dîtin Di gelemperiya gotinên pêşiyan yên Kurdan de zimanekî nêr serdest e. Jina ku li gorî daxwazên mêran û civakê tevdigere û bi weynên xwe yên zayenda civakî radibe li gorî civakê "jina baş" e; jina ku li gorî hest, bîr û baweriyên xwe serbixwe tevdigere û li dijî weynên zayenda civakî tevdigere li gorî civakê "jina nebaş" e.

Peyvên Sereke: Zayenda Civakî, Gotinên Pêşîyan yên Kurdan, Jin, Îmaja Jina Baş, İmaja Jina Nebaş.

\section{GENDER AND GOOD WOMAN-BAD WOMAN PERCEPTION IN KURDISH PROVERBS}

\section{ABSTRACT}

Proverbs are defined as short, meaningful, stereotyped and artistic words that reflect the experience, prejudices and superstitions of the society. It is possible to come across similar stereotypes in all societies and cultures around the world. There are thousands of proverbs on different subjects in Kurdish culture as well. These proverbs also reflect the economic, cultural, social and psychological situation of the Kurds. Gender perception and gender roles play a decisive role in daily life. Business and professional life is shared between women and men according to gender roles and continues traditionally. As in all areas of life, gender roles appear in Kurdish proverbs. A masculine language prevails throughout Kurdish proverbs and the image of good women and bad women is shaped within the framework of the patriarchal approach. This study focuses on the image of good women and bad women in Kurdish proverbs in the context of gender. For this purpose, thousands of verbal and written Kurdish proverbs from different sources were reviewed, and proverbs dealing with gender-based roles of women and men were determined. As a result, women who behave in accordance with the gender roles that society and especially men assign to women have the image of "good woman". Women who oppose the gender roles assigned to them and act according to their individual will, feelings and thoughts have the image of "bad woman".

Keywords: Gender, Kurdish Proverbs, Woman, Good Woman Image, Bad Woman Image 


\section{DESTPÊK}

Çand bi bûyer, dem, serhatî, xebat, afirîneriya hişmendiya kes, kom û civakan ava dibe. Bi rêbazên devkî, nîşandanî, çalakî, berhemî, nivîskî û hwd. ji nifşekî derbasî nifşên din dibe. Her ku dem derbas dibe, serhatî, zanîn û cerebe jî zêde dibin. Bi alîkariya ziman, zanîn û serboriyên hevpar nirx û darazên civakê ava dibin ku em bi giștî wekî bîra çandî dikarin penase bikin. ${ }^{1}$ Bîra çandî, tevî hemû karîgeriyên xwe rê û rêzikên jiyana civakî ava dike. Ev yek jî hîmê folklorê amade dike. Peyva folklorê cara yekemîn di sala 1846an de ji aliyê William John Thoms ve li şûna hêmanên "popular antîkîte" û "popular literature" hatiye bikaranîn. ${ }^{2}$ Bi demê re ji edebiyatê vequetiyaye û wekî zanisteke serbixwe hatiye nirxandin. Folklor, hemû bawerî, rê û rismên civakî, çareserkirina pirsgirêkên tendirustiyê bi rêbazên gelêrî, kar û barên jiyana rojane, xwarin û vexwarin, lixwekirina cil û bergan, berhemên çanda devkî û hwd. di nava xwe dihebîne. Folklor, tevgereke hunerî ye û di demekê de, di nav civakê de xweveber derdikeve holê. ${ }^{3}$

Yek ji binbeşên folklorê çanda devkî, gotinên pêşiyan e. Em teqez nizanin ka gotinên pêşiyan cara yekemîn ji ku derketine û çawa li dinyayê belav bûne. Lê em ê li vir mînakekê bidin. Di vê mijarê de pisporê gotinên pêşiyan Wolfgang Mieder angaşteke derdixe pêş. Li gorî wî, gotinên pêşiyan li parzemîna Ewropa derketine û piştre li dinyayê belav bûne. Çar jêderên gotinên pêşiyan hene: Di rêza yekemîn de serdema Roma û Yewnaniya kevn heye ku bi zimanê latînî dihate axaftin. Pêşengê wê jî feylesofê Yewnanî Arîsto ye. Di rêza duyemîn de Pirtûka Pîroz/Mizgînî (Încîl) heye. Rêza sêyemîn latîniya qirnê navîn ku wekî frensiya kevin tê zanîn û di rêza dawî de jî metnên nûjen hene. ${ }^{4}$ Teqez angaştên din jî hene lê ev yek mijara xebateke din e. Jêder çi dibe bila bibe gotinên pêşiyan li her devera dinyayê bala lêkolîner û zanyaran kişandiye. Li welatên pêşketî, xebatên li ser gotinên pêşiyan wisa kûr û zêde bûne ku tenê li ser gotinên pêşiyan du zanist ava bûne. Yek jê "paramîyografî" ye ku bi tomarkirin û parastina gotinên pêşiyan ve mijûl e. Ya din jî qada lêkolînên gotinên li ser pêşiyan daye ber xwe. Herweha zanisteke din jî heye ku jê re "frazelojî" dibêjin. Ew jî, gotinên pêşiyan jî di nav de, hemû cureyên edebî yên hevokî dide ber xwe. Wekî, gotinên pêşiyan, biwêj, gotinên kûrwate, zûgotinêk, dubare û hwd. ${ }^{5}$

Dema ku em li lêkolînên derbarê gotinên pêşiyan de hûr dibin, em dibînin ku lêkolînên derbarê gotinên pêşiyan yên Kurdan hîn di asta berhevkirinê de ne. Wekî mînakekê em dikarin behsa Law Reşî̀ û Hîlmiyê Siwêregî bikin. Her du, hîn salên 1918 û 1919an de bi awayekî zanistî li ser gotinên pêşiyan yên Kurdan hûr bûne û hewldaneke dabeşkirinê kirine. Law Reşîd, di kovara Jînê de van gotinên pêşiyan li gorî mijaran wekî gotinên civakî, lehengî, edebî/wêjeyî, darazî, bûyerî, navmalî, çandinî, rewşa hewayê, dîrokî, efsanewî, kenokî û kompozîsyonî (çîrokî) dabeş kiriye û digel tirkiya wan li ser wateya wan rawestiye. ${ }^{6}$

Gotinên pêşiyan, di ferhenga Oxfordê de wekî "hevok ango gotina nav û deng ku şîretekê, darazekê, tecrûbeyekê destnîşan dike" hatiye penasekirin. ${ }^{7}$ Di Ferhenga Çandî ya Emerikî de, wekî "Gotinên kurt û watedar ku cerebeya dariştî, pêşdaraz û baweriyên batil ên civakan destnîşan didin." hatiye penasekirin. ${ }^{8}$ Pisporê Emerîkî yê gotinên pêşiyan Bartlett Jere Whiting, ji bo penasekirina gotinên pêşiyan ji taybetiyên

1 - Jan Assmann, Kültürel Bellek, çev. Ayşe Tekin, (İstanbul: Weşanên Ayrıntı, 2001), 140.

2 - Richard M. Dorson, W.J. Thoms, "William John Thoms ve "Folklor” Başlıklı Yazısı”, çev. Serpil Aygün Cengiz, ed. M. Öcal Oğuz, Selcan Gürçayır, Halk Biliminde Kuramlar ve Yaklaşımlar 2, (Ankara: Weşanên Geleneksel, 2003 ), 5.

3 - Dan Ben AMOS, "Şartlar ve Çevre İçinde Folklorun Bir Tanımına Doğru”, çev. Metin Ekici, ed. M. Öcal Oğuz, Metin Ekici, Nebi Özdemir, Gülin Öğüt Eker, Selcan Gürçayır, Halk Biliminde Kuramlar ve Yaklaşımlar 1, (Ankara: Weşanên Geleneksel, 2006), 47.

4 - Wolfgang Mieder, Proverbs Speak Louder Than Words: Wisdom in Art, Culture, Folklore, History, Literature and Mass Media (New York: Peter Lang press, 2008), 14.

5 - Noor Sanauddin, School of Social and Political Sciences, (Glasgow: University of Glasgow, The College Social Sciences, PhD, 2015), 17.

6 - Law Reşîd, "Kürdlerde Durub-i Emsal”, Wergêr ji tîpên erebî bo tîpên latînî: Mehmed Emin Bozarslan, Jîn, Kovara Kurdî-Tirkî, Kürtçe- Türkçe Dergi, ed. Mehmed Emin Bozarslan, (Uppsala: Weşanxana Deng, 1985), 264.

7 - Oxford Learners Dictionaries, "Proverb”, (29 Çile 2021).

8 - E. D. Hirsch, Joseph F. Kett, James Trefil, The New Dictionary of Cultural Literacy, (Boston, New York: Houghton Mifflin Company, 2002), "Proverb", 47. 
wan sûd werdigre û dibêje ku "Gotinên pêşiyan, ev gotinên zelal ku rasteke ango rastiyekê bi hunera alîterasyonê û serwayê hatine xemilandin in. ” Piştre Whiting, hinekî din li ser taybetiyên gotinên pêşiyan hûr dibe û hin regezên din jî diyar dike. Dibêje, divê digel wateyên wan ên rastî divê wateyeke mecazî jî bihebînin, ji aliyê gel ve hatibin pejirandin, şopa ji demên bihurî li ser wan be û di dem û cihên cûda de hatibin pesendkirin. ${ }^{9}$ Li gorî Arewa û Dundes, gotinên pêşiyan rewşekê kurtebir dikin, darazekê pêşniyar dikin û ji bo têgihiştina rewşa heyî mînakan ji serboriyên berê didin. ${ }^{10}$

Berhevkar û nivîskarê Kurd, Çiya Mazî gotinên pêşiyan, wekî "Hevokên darazî, hukmî û bi pendî ne ku li nava gel ji bo vegotin û rexneya tiştekî, bûyerekê an ramanekê hatine gotin" penase kiriye. ${ }^{11}$

Wekî ji penaseyan jî tê fêmkirin, gotinên pêşiyan ne agahî û zanînên mayinde ne; li gorî dem, çand, ziman û civakan diguherin. Li gor pêwîstî û hevcedaritên çanda devkî teşe digrin. Ji bo ku veguhastina ji nifşên nû re hêsan be, gotin bi awayekî kurt ku di hişê mirovan de bimîne hatine gotin. ${ }^{12}$ Gotinên pêşiyan rewşa hişmendiya civakê nîşan didin û pêwistiyên civakê jî rawe dikin. Wek mînak, di Kurdî de "Mala ku goșt bikeviyê, derd nakeviyê"'13, di tirkî de "Güneş girmeyen eve doktor girer," "An apple a day keeps the doctor away"15 (Rojê sêvek ji dixtoran dûr dihêle). Her yek ji van gotinên pêşiyan ji bo tendirustiya mirovan tiştekî pêşkêş dike. Tê zanîn ku di nav gelek neteweyan de gotinên pêşiyan ên hevpar ango nêzikî hev jî hene. Wek mînak, "Malê dinyê qirêja destan e."16; "Dünya malı dünyada kalır." 17 (Malê dinê li dinê dimîne-Gotina pêşiyan a tirkan); "You can't take it with you when you die." "I8 (Dema ku tu mirî, tu nikarî tiştekî bi xwe re bibî-gotina pêşiyan a îngîlizan). kêm-zêde di heman wateyê de ne.

Ji aliyê teşe û wate ve taybetiyên gotinên pêşiyan jî hene.

Gelek gotinên pêşiyan dijwate ne. Wekî: "Jin, taca zêrîn e." ${ }_{19} \hat{u}$ "Jin û derew mirovên (yek)hev in. "20

Gotinên kurt, zelal û watedar in. Wekî: "Mal bi xwedî" (mal bi xwedî dibe mal), "dûr be nûr be", "hindik, rindik". ${ }^{21}$

Di gotinên pêşiyan de wateya mecazî ji ya rastî pêştir e. Mînak, "Çerçî helawa xwe naxwe." "22, "Donê nemerdan li ser nanê nemerdan. "23

Di gotinên pêşiyan de hunera şibandinê gelekî zêde ye. Mînak, "Jin zeviya ber gund e."24, "Jin û mêr tevr û bêr e." "25, "Dara bî weke jina bî.",26

9 - Sarwet Rasul, "Gender and Power Relationships in the Language of Proverbs: Image of a Woman", FWU Journal of Social Sciences, 9/2 (Winter 2015), 52.

10 - E. Ojo Arewa, Alan Dundes, "Proverbs and the Etnograpy of Speaking Folklore”, American Anthropologist, 66/6 (1964), 70-85.

11 - Çiya Mazî, Ferhenga Gotinên Pêşiyan, (Stenbol: Weşanên Elma, Sibat 2005), 8.

12 - Necat Keskin, Folklor û Edebiyata Gelêrî,, (Stembol: Weşanên Avesta, 2009), 298.

13 - J1, Hevpeyvîna rû bi rû, 19 Çile 2021.

14 - Güncel Türkçe Sözlük, https://sozluk.gov.tr/

15 - Cambridge Dictionary, https://dictionary.cambridge.org/tr/s\%C3\%B6zl\%C3\%BCk/ingilizce/an-apple-a-day-keeps-thedoctor-away 02.05.2021.

16 - Mazî, Ferhenga Gotinên Pêşiyan,292.

17 - Türk Dil Kurumu Sözlükleri, https://sozluk.gov.tr/ 12.06.2021.

18 - Cambridge Dictionary, https://dictionary.cambridge.org/tr/s\%C3\%B6zl\%C3\%BCk/ingilizce/an-apple-a-day-keeps-thedoctor-away 02.05.2021.

19 - Mehmet Oncu, Di Gotinên Pêşiyan de Jin, (Van: Weşanên Sîtav,2020), 131.

20 - Mehmet Oncu, Gotinên Pêşiyan, (Stenbol: Weşanên Enstitûya Stenbolê, 2009), 360.

21 - J2, Hevpeyvîna rû bi rû, 20 Çile 2021.

22 - Oncu, Gotinên Pêşiyan, 109.

23 - Oncu, Gotinên Pêşiyan, 190.

24 - Oncu, Gotinên Pêşiyan, 359.

25 - Mazî, Ferhenga Gotinên Pêşiyan,242.

26 - Oncu, Gotinên Pêşiyan, 127. 
Hin gotinên pêşiyan dirêj û çîrokî ne. Mînak: “Kul hate malê. Got: 'Ez li serê sê kesan.’ Tevan li çavê xulam nihêrî. Xulam got: 'Yek ez, lê yên mayîn kî ne?"'27

Hin gotinên pêşiyan bi demê re nirx û wateya wan wenda dibe ango gelekî kêm dibe. Mînak: "Ji milkan bikire aş, ji huneran hu bike simteraş" ${ }^{28}$ Simteraş, nalbend e. Wer xuya ye ku demekê dahata aşvanî û nal bendiyê zêde bûye û ji ber vê yekê jî her du huner di rewacê de bûne.

Piranî bi serwa (hevdeng-qafiye) û redîf in. Mînak: "Zêr zane, zor zane, devê tivinga mor zane. ${ }^{29}$ ("zane" redîf; "r"jî serwa ye.)

Hin gotinên pêşiyan hene ku ji aliyê hevoksazî û rastnivîsê ve kêm an şaş in. Sedemên vê yekê dido ne. Yek jê bandora zimanê devkî û devokê ye, ya din jî xema serwa û ahengê ye. Lê ya girîng ew e ku bi vê rêbazê gotinên pêşiyan kurt dibin û bi hêsanî têne jiberkirin. ${ }^{30}$ Di gelek gotinên pêşiyan de hunera alîterasyonê ango bikaranîna hemdengiyê heye. Wekî mînak, di gotina pêşiyan a "Heft kilamên hirçê hene; her heft jî li ser dara hirmê ne." 31 de ji neh bêjeyan şeş bêje bi tîpa "h" dest pê dikin.

Gotinên pêşiyan, ji dergûşê heta bi piştî mirinê li ser her kesî, her statûyê (wekî hevser jin û mêr, dayik, damarî, jinebî, xal, ap, xwarzê, kal, pîr, axa, xulam û hwd.), her rewşa xwezayê û taybetiyên mirovan (wekî hesûdî, camêrî, nemerdî, bênamûsî, dizî, şêlandin, wekhevî, neyartî, zordestî, biratî û hwd.) hatine gotin. Derfetên vê gotarê rê nadin ku em hemû cureyan binirxînin. Helbet bi dehan gotinên pêşiyan ên Kurdan kul i ser wekheviya jin û meran hûr dibin jî hene. Lê belê di vê xebatê de mebesta me ew e ku piştî penaseyeke kurt û vegotina hin taybetiyên gotinên pêşiyan, di çarçoveya weynên zayenda civakî de li ser gotinên pêşiyan yên Kurdan hûr bibin û îmaja jina baş û nebaş derxînin.

Derbarê gotinên pêşiyan de gelek xebatên berhevkariyê hatine meşandin. Tê zanîn ku hîn di salên 1918 û 1919an de nivîskar û lêkolînerên Kurd Law Reşid û Hîlmiyê Siwêregî li Stenbolê di weşanên kurdî-tirkî de cih dane gotinên pêşiyan û wateyên wan. Li gorî lêkolînên Mustafa Öztürk, rêzegotarên van her du nivîskaran wekî xebatên herî pêşîn yê vê qadê ne. ${ }^{32}$ Lê tê zanîn ku Mela Mehmûdê Bayezîdî hîn di nîveka sedsala 19an de bi navê "Kilam, Berîte, Gotinên Pêş̧iyan" berhemek nivîsandiye. ${ }^{33}$ Celadet Elî bedirxan, li Şamê di nav rûpelên Hawarê de cih daye xebatên ziman û çanda Kurdan û herweha pirsgirêkên civakî. Di gelek cureyên edebiyata modern de berhem nivîsandine, werger kiriye û cih daye nimûneyên edebiyata gelêrî. Di hejmara pêşî de tê eşkerekirin ku dê Hawar ji bilî siyasetê bi her tiştê têkilî kurdî kurdayetiyê bike mijara weşana xwe. ${ }^{34}$ Girêdayî vê mijarê hêjayî gotinê ye ku bi sedan gotinên pêşiyan yên Kurdan digel wate û şîroveyên wan wergerandiye ser zimanê frensî. ${ }^{35}$ Herweha derbarê berawirdkirina gotinên pêşiyan de gelek xebat hene. Wek mînak, Karacan û Erdinç, gotinên pêşiyan ên Kurdî û Rusî ji aliyê peyv û wate ve dane ber hev û gihaştine wê encamê ku gelek gotinên pêşiyan ên hemwate ango nêzwate di herdu zimanan de jî hene. Mebesta gotinên pêşiyan, dûrketina ji rêya xerab û bervêçûyîna rêya rast û baş e. ${ }^{36}$

27 - Oncu, Gotinên Pêşiyan, 434.

28 - Oncu, Gotinên Pêşiyan, 351.

29 - Mazî, Ferhenga Gotinên Pêşiyan, 425.

30 - Hasan Karacan, İhsan Ayus, “Kürtçe ve Türkçe Atasözlerinin Karşılaştırmalı Bir Biçimde İncelenmesi”, ed. Hasan Karacan, Kürdoloji Akademik Çalışmalar 1 (Diyarbakır: Dicle Üniversitesi Basımevi, 2016), 115.

31 - J3, Hevpeyvîna rû bi rû, 21 Çile 2021.

32 - Mustafa Öztürk, "Vekolînek Derbarê Gotinên Peşiyan Ên Kurmancî De”, Mukaddime, 2015, 6/2, 333.

33 - Kadri Yıldırım û hwd., Ji Destpêkê Heta Niha Folklora Kurdî (Stenbol: Weşanên Enstitûya Zimanên Zindî ya Zanîngeha Mardin Artuklu, 2013), 54.

34 - Tekin Çifçi, “Kültür, Sanat ve Siyaset Adamı Olarak Celadet Ali Bedirhan”, Kürdoloji Akademik Çalışmalar 2, ed. Hasan Karacan, (Ankara: Yargı Yayınevi, 2016), 509.

35 - Mihemed Bekir, Hawar, (Amadekar), (Stockholm: Weşanên Nûdem, 1987).

36 - Hasan Karacan, Recep Erdinç, "Rus ve Kürt Atasözlerinin Kelime ve Anlam Karşılaştırması”, Dicle Üniversitesi İlahiyat Fakültesi Dergisi,16/2, (2014), 582. 


\section{ZAYENDA CIVAKÎ Û ÎMAJA JiNA “BAŞ” -“NEBAŞ” Di GOTINÊN PÊŞIYAN DE}

Zayenda civakî, wek têgeh weyn (rol), berpirsiyarî û taybetiyên kesayetiyê ku ji aliyê civakê ve hatine destnîşankirin diyar dike. Zayenda civakî, ji cudatiyên bîyolojîk (laşî) dernaxe pêş. Bêtir li ser çawaniya têgihiştin û rawekirina tevgera jin û mêran a ku ji aliyê civakê ve tê hêvîkirin radiweste. Weyna zayenda civakî ango rewşa jinbûn û mêrbûnê, tevî karîgerên din ên civakîbûnê pêvajoyeke hînbûnê ye. Ev hînbûyîn, li gorî demê û çandê diguhere. Ji nav malê dest pê dike, li dibistanan, li derve, li saziyan û kargehan didome. Her yek ji van qadan di afirandin û jinûveteşedana zayenda civakî de bi bandor e. Lewra, berdewamiya karîgerî û cudatiyên zayenda civakî bi saya serê van saziyan pêk tê. ${ }^{37}$ Çanda civakî, hîmê raman, kar, berpirsiyarî û tevgerên jin û mêran datîne. Li gorî hişmendiya zayenda civakî ya mêran debara malê berpirsiyariya mêran e. Herweha mêr ji xweykirin û parastina jina xwe jî berpirsiyar e. Anîn, xweykirin, mezinkirina zarokan û sazkirin û birêvebirina kar û barên nav malê jî berpirsiyariya jinan e. ${ }^{38}$

Digel hin îstîsnayan piraniya qadên jiyanê li gorî pergala pederşahiyê û weynên zayenda civakî hatine sererastkirin. Ev pergal, herwekî kar û xebatên rojane di pîşeyan de jî xûya dibe. Digel ku zagoneke fermî di vê mijarê de nîn e jî hemû kar û pişse -bi perdeyeke xeyalî- wekî "karên jinan" û "karên mêran" bûne du tebeqe. Ji vê yekê re "çînîbûna berwar (asoyî)" tê gotin. Herweha di nava jin û mêrên heman pîşeyê de jî "çînîbûneke stûnî (berjor)" heye. Wek mînak mêr di heman pîşeyê de wekî serhosta, şef û rêvebir di payeyên bilind de wekî "pistokspî" 39 dixebitin. Lê belê jinên ku di heman pîşeyê de bi mehaneyên (meaş) kêm di karên ji rêzê wekî "pistokşîn" ${ }^{40}$ dixebitin. ${ }^{41}$ Em vê dabeşkirinê di gotinên pêşiyan yên Kurdan de jî dibînin. Xebatên bîrdoziyên zayenda civakî ku li ser zimanê gotinên pêşiyan hatine kirin diyar dikin ku di xwezaya gotinên pêşiyan de dubendiya zayenda civakî heye. Digel ku piştî têkoşînên jinan hinekî kêm têne bikaranîn jî hebûna xwe didomînin..$^{42}$ Gotineke pêşiyan a Kurdan heye. Dibêje: "Jin, jin e; mêr, mêr e. " ${ }^{43}$ Bi vê gotina pêşiyan kar û berpirsiyariyên jin û mêran teqez ji hev hatine qetandin. Jin wek avakarê malê, kebaniya malê, wek dîwarê malê tê pêşkêşkirin. Wekî, "Kebanî jî pê re dikele heta girar dikele." "44 Wate: Heta karê malê neqede jin nikare terka malê û şîvê bike. "Jin dîwarê hundir e, mêr dîwarê derve ye. "45 Wate: Jin ji karê nav malê re jêhatî ye; mêr jî ji karên derve re.

Ji aliyê zayenda civakî ve bi giştî du taybetiyên gotinên pêşiyan hene:

Ya pêşî, digel hin îstîsnayan gotinên pêşiyan berhemên civakên pederşahiyê ne û bi devê mêran hatine gotin. Ya duyemîn jin, wekî heyberên neyînî di gotinên pêşiyan de cih digrin. Jin û jinbûnî tê piçûkxistin, kêmdîtin. ${ }^{46}$

Hin gotinên pêşiyan hene ku heqaretê li kesayetiya jinan dikin û wan piçûk dixin. Wek mînak, “Jinikê got mela; "mehra min mehkem nebire, ez dest-pê nesekinî me." 47 "Dersê digire ji jinê, dihere dibe dijminê" 48 Wate: Kesê ku bi aqilê jina xwe bimeşe dê gelek şaşitiyan bike û xelkê ji xwe re bike neyar.

37 - Sema Çifçi, Vasfiye Bayram Değer, Nurcan Bayram, “Üniversite Öğrencilerinin Toplumsal Cinsiyet Algıları: Mardin Artuklu Üniversitesi Örneği”, Uluslararası Hakemli Ekonomi Yönetimi Araştırmaları Dergisi, 14 (2017), 38.

38 - Gary N. Powell, Jeffrey H. Greenhaus, "Sex, gender, and decisions at the family-work interface”. Journal of Management, 36/4 (2010), 1011-1039.

39 - Pistokspî: Ew kesên ku bi hêza mêjî û zanistê dixebitin û meaşeke/dahateke baş distînin.

40 - Pistokşîn: Ew kesên (karker) ku bi hêza laşî dixebitin û meaş ango yewmîyeya kêm distînin.

41 - İnci Parlaktuna, “ Türkiye’de Cinsiyete Dayalı Mesleki Ayrımcılığın Analizi”, Ege Akademik Bakış Dergisi, 10/4 (2010), 9.

42 - Benedicta Adokarley Lomotey, Sewoenam Chachu, "Gender ideologies and power relations in proverbs: A cross-cultural study", Journal of Pragmatics, 2020; 168: 70. (69-80).

43 - Oncu, Di Gotinên Pêşiyan de Jin, 120.

44 - Oncu, Di Gotinên Pêşiyan de Jin, 193.

45 - Oncu, Di Gotinên Pêşiyan de Jin, 112.

46 - Liisa Granbom-Herranen, "Women's Place in Finnish Proverbs from Childhood”, Folklore: Electronic Journal of Folklore, 46 (2010), 95-110.

47 - Oncu, Gotinên Pêşiyan, 366.

48 - Mustafa Borak, Ferhenga Biwêjan, (Stenbol: Weşanên Enstitûya Stenbolê, 2005). 
"Jin bimire, mêr jar dibe; mêr bimire jin har dibe." ${ }^{49}$ Wate: Dema ku jin bimire, mêr nikare karê nav malê û nan û şîva xwe amade bike. Ji ber vê yekê jî jar-zeîf dibe. Lê heger mêr bimire, jin bê serî dimîne û ji ber vê yekê har dibe. Ev "harbûn" hem di wateya zayendî hem jî di wateya tevgerên bêqayîde de ye.

Li aliyê din em dibînin ku jin jî dixwazin bikevin bin baskên mêran. Wekî,

"Bila jina mêran bî, li ber stêrk $\hat{u}$ bîyûran bî"," Wate: Heger mêr yekî li gorî dilê jinê be, jin dikare li hemberî hemû pirsgirêkan li ber xwe bide.

"Darê bi tenê mîna mêrê li ber hikmê jinê, darê dido agirê Kodo, darê sisê agirê lîsê, darê pênc û şeş agir dike xweş. " ${ }^{51}$ Wate: Digel ku ev gotina pêşiyan li yekitiyê hatiye gotin jî tenêtî wekî bindestiya jinan hatiye pêşkêşkirin.

"Mêrê xeberjin, berxê ber bizin, şûva bi kizin tu tişt jê dernayê"52 Wate: Mêrê ku li gorî axaftina jina xwe tev bigere tu bi ser nakeve. Lewra, jin rêya rast nizanin tenê mêr dikarin biryarên di cih de bidin.

Hin gotinên pêşiyan hene ku jin û mêran dixin nav qaliban. Gelek liv û tevgerên asayî û rojane layiqî wan nabînin. Çend mînakên van gotinan weha ne:

"Fediyokî li mêran, kenokî li jinan nayê." ${ }_{53}$

“Jina şermîn bi şarekî, mêrê şermîn bi karekî." ${ }_{54}$

"Jina bê heya, wek girara bê xwê ye" "W5 Wate: Şerm ji bo jinan pêwîst e. Jina bi fedî bi qîmet e. Mêrê bi fedî bêqîmet e.

Gelek tebîet û xûyên baş hene ku wan dispêrin mêran û mêraniyê. Wek mînak, di çanda Kurdan û gelek civakên pederşahî de şer û kuştina mirovan wekî karê mêran tê dîtin. Li gorî weyna zayenda civakî, jin bêzirav in, nikarin bi kûştina mirovan rabin. Helbet şer û pevçûn û kuştin jî li gorî şert û mercan pêk tê. Ew yek ji bo çanda Kurdan jî weha ye. Çend mînakên vê mijarê weha ne:

Di gelek gotinên pêşiyan de di navbera wekî zayend nêrbûn û wekî weyna zayenda civakî mêrbûnê de têkilî hatiye danîn. Wek mînak, di gotina pêşiyan a "Berxê nêr, ji bo kêr" ${ }_{56}$ de "berxê nêr" mêraniyê û "kêr" jî mirin û kuştinê temsîl dike. Li aliyê din ev gotina pêşiyan bûyera Hz. Îbrahîm ku kurê xwe -Hz. Îsmaîlwekî qurban pêşkêş dike tîne bîra mirovan. ${ }^{57}$ Çi gotina pêş̧iyan dibe, çi jî ev bûyer dibe. Em têdigihên ku lawik ango zarokên kur ji piçûkantiyê de ji bo şer têne amadekirin.

"Şêr bi lepê xwe, mêr bi destê xwe." ${ }^{8}$ Wekî ji vê gotinê jî fêm dibe. Di gelek gotinên pêşiyan de mêran dişibînin sewal û heyberên xûrt û mezin. Şêr, di nava lawiran de bi hêza xwe bi nav û deng e. Li gorî baweriya gelêrî hêza mirovan jî ji mêran xûya dibe.

"Sebr sekna mêran e." ${ }_{59}$ Hal ev e ku sebir, ji bo her kesî gelek caran tevgereke baş e. Ji ber ku derfeta aramiyê û ramanê dide mirovan. Ew pîleya çêkirina xeletiyan û tevgerên şaş kêm dike. Lê em dibînin ku vê tevgera baş dixin para mêran.

49 - Oncu, Gotinên Pêşiyan, 358.

50 - Law Reşîd, “Kûrdlerde Durub -i Emsal”, 301.

51 - Cewerî, Hawar 2, 1032.

52 - Hin Gotinên Pêşiyan, https://candname.com/gotinen-pesiyan-3/ 12.06.2021.

53 - Oncu, Di Gotinên Pêşiyan de Jin, 77.

54 - Oncu, Di Gotinên Pêşiyan de Jin, 157.

55 - Mazî, Ferhenga Gotinên Pêşiyan, 243.

56 - Oncu, Gotinên Pêşiyan, 47.

57 - Tekin Çifçi, Baweriyên Gelêrî û Dermanê Kurmancî Li Herêma Semsûrê, (Diyarbakır: Zanîngeha Dîcleyê, Enstitûya Zanistên Civakî Makezanista Ziman û Çanda Kurdî, Teza Doktorayê, 2019), 84.

58 - Mazî, Ferhenga Gotinên Pêşiyan, 375.

59 - Oncu, Gotinên Pêşiyan, 596. 
"Mêr, mêran nas dike; qûş paldimê nas dike $60 "$

"Mêrê kâmil, di paçikê çêl de xuya ye." ${ }_{11}$

Wate bi giştî li ser kesê jîr û zana ye ku hîn di piçûkantiya xwe de remzên wan kifş dibin û bal dikşinin ser xwe. Kê belê di vê gotinê de jî başî nîspet bi mêran ve ye. Behsa jinan nabe.

"Mêrê kê pir bin, ew mêr e. " ${ }^{2}$ Ew gotin jî cardin dide diyarkirin ku hêza malbat û eşîran bi hejmara mêran tê pîvan.

"Mala mêran, kaniya zêran." ${ }_{63}$ Wate: Mala ku mêr tê de hebin û kedkar bin, dahata wan zêde ye. Di geşkirina rewşa aborî ya malbatê de weyna jinê tüne tê hesibandin. Ew yek berpirsiyariya meran jî zêde dike. Wekî dibêjin,

"Mirovê tirsonek her dimre, ê mêr carkê." "64

"Bixwe bi gurikî, rabe bi mêrikî." ${ }^{5}$ Di vê gotinê de jî xwarin û xîreta nîsbet bi mêran ve tê kirin. Wekî ji mînakan xûya dibe hêz, lehengî û wêrekî bi mêran ve hatiye girêdan. Berevajî vê yekê liv û tevgerên ku qelsiyê, tebîetên nebaş, hestiyariyê û tirsê nîşan didin jî bi jinan ve hatine girêdan. Wekî,

"Qehbê to çima ber nagirî?? Got: Heta êk çêdikit dek xirab dikit." "66

“Jin dara şikestî ne. " 67 Wate: Jin nazik in, kêm in, qels in.

“Gavan hat zeviye, jina gavan nu bezî teşiye. "68 Wate: Jin, teral in lê xwe wekî jêhatî nîşan didin.

"Bi mineminê mirov xelas nabe ji destê jinê" 69 Wate: Jin, zêde lê vala xeber didin. Mêr tenê bi zextê dikare zora jinê bibe.

"Bi lavlavê, mirov ji ber jina xwe nake der."70

"Heft jinen eşî̂ê rahêjane barekî hiriye, gotine: "Wey li me bêkesan!" '11 Wate: Jin, ji aliyê hêz û quwetê ve qels in.

"Berdana jinê edeta dinê""72 Di vê gotina pêşiyan de îradeya jinan tune tê hesibandin. Li gorî kevneşopî û weynên zayenda civakî "mêr" dikare jinê berde; "jin" nikare dev ji mêrê xwe berde. Lewra negotine, "Mêrberdan edeta dinê"

Mêr û jin di zewacê girîngiyê didin temen. Lê mêr, bêtir girîngiyê didin bekaretê. Ji ber vê yekê jî gotine:

"Bila keç be, bila pîr be." "73

"Li rîya here bila dûr bit, li borîya here bila kûr bit, keça bîne bila pîr bit." 74

60 - Mazî, Ferhenga Gotinên Pêşiyan, 298.

61 - Oncu, Gotinên Pêşiyan, 488.

62 - Oncu, Gotinên Pêşiyan, 488.

63 - Mazî, Ferhenga Gotinên Pêşiyan, 290.

64 - Tawûzparêz (C. Elî Bedirxan), "Proverbes”, Hawar, Amadekar: Firat Cewerî, (Stockholm: Weşanên Nûdem, 1998), 1050.

65 - Mazî, Ferhenga Gotinên Pêşiyan, 114.

66 - Mele Mehmûd Dêrşewî, Miştaxa Çiya Ji Gotinên Pêşiya, wer. Serbest Zaxoyî, (Stockholm:1989), 213.

67 - Oncu, Di gotinên Pêşiyan de Jin, 109.

68 - Dêrşewî, Miştaxa Çiya Ji Gotinên Pêşiya.

69 - Oncu, Di gotinên Pêşiyan, 60.

70 - Cewerî, Hawar 2, 1031.

71 - Oncu, Di gotinên Pêşiyan de Jin, 90.

72 - Mela Mihyedîn, “Di Gotinên Pêşiyan de Jin -1”, https://candname.com/di-gotinen-pesiyan-de-jin-1/ 12.07.2021.

73 - Oncu, Di gotinên Pêşiyan, 73.

74 - Dêrşewî, Miştaxa Çiya Ji Gotinên Pêşiya, 174. 
Wate: Rê çiqas dûr be jî ji xweqelibandin û xweşaşkirina li ser rêyên ku mirov nizane çêtir e. Borî, deverên ku berê hatine ceribandin in. Mirov di ser wan re derbasî aliyê din dibe. Ango tiştê ceribandî ye, gûmana têkçûyînê kêm e. Herweha, di zewacê de temen çiqas mezin dibe bila be ya girîng keçikbûn e. Ji ber ku li gorî baweriya civakê, keçik wekî jinebîyan ne fêlbaz û davdoz in. Jinebî wekî roviyê feltînok in.

Gelek gotinên pêşiyan hene ku jinê tebatî (pasif/sist) dibînin. Di pêvajoya biryara zewacê de kes bi jinê naşêwire. Jin wekî berdêla xwînê, berdêlî ango ji bo xatirê qelen û berjewendiyên din bê dilê wan têne zewicandin. Ew yek jî rê li ber bêdadiyan û zordestiyê vedike. Ev pirsgirêk di gelek gotinên pêşiyan de eşkere tê gotin. Wekî,

"Bûk li ser hespê ye, kes nizane kismetê kê ye."75

"Mêr li ku, jin li wir gereg e." ${ }^{76}$ Cardin jiyana jinan bi jiyana mêran ve tê girêdan.

"Mala bê ga, bi golik nabe." "77 Wate: Ji malekê re mêr pêwîst in. Mala ku tê de mêr tuneye nijada wan berdewam nake.

Di gelek gotinên pêşiyan de jin û mêr ji aliyê laşî û xweşikbûnê ve duçarî dubendiya zayenda civakî dibin. Wek mînak;

"Mêr bi mûyê xwe, jin bi rûyê xwe." "78 Wate: Xweşikbûna jinan bi rûyê wan; ya mêran jî bi simbêl û mûyê ser singa wan xûya dibe.)

“Jin bi kofî û kitan. "79 Wate: Li gorî kevneşopî weyna zayenda civakî jin, heger serê wan girtî be xweşik in.

Xweşikbûna jinê ji bo mêran pirsgirêkeke mezin e. Xweşikiya jinê bi xwe re hesûdiya mêran jî tîne. Wekî mînak,

“Jina çist çavêş $e$, jina bedew dilêş e." "80 Wate: Jina ku ne xweşik ve tenê dîdariya mêrê xwe xera dike. Lê belê heger xweşik be dê her kes lê binihêre ew jî dê bibe kul û êş di dilê mêrê wê de. Jina nexweşik ango biqusûr carinan çêtir e. Wekî,

"Ji milkan aş, ji jinan jinen şaş, ji mêran mêrên ne bide pêş, ne bide paş. "

"Jina spehî her bela ye." "W2 Wate: Jina xweşik, her kes lê dinihêre. Ew jî rê li ber hesûdiya mêrê wê vedike. Heger jin xweşik be, dê mêrên xerîb bi dora wê bikevin. Mêrê wê û merivên wê jî vê yekî qebul nakin û dê bi wan kesên ku li jinê dinihêrin re şer bikin. Ew jî her serêş e. Ji ber ku mêr, parastina jinê wekî namûs dihesibînin. Destdirêjiya namûsê jî li gel mêrê Kurd nayê pejirandin. Tê zanîn ku tenê ji ber vê hişmendiyê gelek şer û pevçûn çêdibin û mirov têne kuştin.

Gelek caran ji bo mêran çelengî û lihevhatîbûn ne girîng e, Wekî,

"Mêr mêr e, çi kulekî, çi kor, çi kerr e." 83

"Mêr mêr e; çi kulek, çi nabîna, çi kerr e."

Jin, di nav malê de wekî keç, bûk, jin (hevser), dayik, damarî (jinbav), jinebî û pîrik bi weynên zayenda civakî ve radibin. Di gelek gotinên pêşiyan de pesnê jina tê dayîn. Ji ber ku ew, bi serfirazî berpirsiyariyên xwe yên weynên zayenda civakî bi cih tînin.

75 - Mehmet Güzeler, Folklora Qerejdaxê, (İstanbul: Weşanên Nûbihar, 2021), 32.

76 - Oncu, Gotinên Pêşiyan, 486.

77 - Oncu, Gotinên Pêşiyan, 462.

78 - Mazî, Ferhenga Gotinên Pêşiyan, 298.

79 - Oncu, Gotinên Pêşiyan, 361.

80 - Oncu, Gotinên Pêşiyan de Jin, 147.

81 - Mazî, Ferhenga Gotinên Pêşiyan, 237.

82 - Oncu, Gotinên Pêşiyan, 364.

83 - Oncu, Gotinên Pêşiyan, 486.

84 - “Gotinên Pêşiyan”, https://www.bernamegeh.org/2019/08/02/kurtce-atasozleri-gotinen-pesiyan/ 12.08.2021. 
"Keça malan, bûka xalan." 85

"Bûka malê rewşa malê." "86

"Bûka çê delala malê." " 87

"Jin gol e, mêr çol e." 88 Wate: Mêr li derve dixebite lê ya girîng ew e ku jin li malê berhev dike.

Lê gotinên li dijî van darazan jî gelek in. Di van gotinên pêşiyan de em gazin û rexneyan dibînin. Bi taybetî gazin ji xesû, hewî, damarî û jinebiyan dibin. Em dikarin çend mînakên vê yekê weha bidin:

"Jinbavê jina bavê, birçî me ber dolabê" "89 Wate: Dêmarî nikare wekî diya zarokan li wan miqate bibe. Dema ku diya zarokan tunebe, ew li benda rehma dêmariya dimînin û şerpeze dibin.

"Ku dê bû dêmarî bav î berê bavmarî ye." "90

"Dê ku bû dêmarî, bav dibî filê Kavarî̀" (Her du gotin jî di heman wateyê de ne. Wate: Piştî ku zarok ketin ber destê dêmariyan, bavê wan wekî xerîbekî tevdigere. Lewra, jina nû rê nade ku bav bi berpirsiyarî, weynî û peywirên xwe bi cih bîne.)

"Gazinê bûkê ji xesûyê ye."

"Gotin birayê te bû axa, got jina wî bû xatûn." 93 Wate: Piştî ku mêr zewicî malbata wî ji hebûn û dewlemendiya wî nikare sûdê werbigre. Êdî ew ji bo jina xwe û malbata xwe dixebite.

Keda jinê ku li malê tê dayîn ji aliyê mêr û civakê ve nayê dîtin. Wekî gotine:

"Jinan zehmetî kişand, dia li mêran kirin. "94 Ev gotina pêşiyan bi du awayan dikare were şîrovekirin lê encam naguhere. Ya yekemîn, jin li malê çiqas bixebite û rewşa malê baş bike, dibe serbilindiya mêrê wê. Gel pesnê mêrê wê dide. Dibêje, "Mala filankesî çi mal e, helal be jê re." Ya duyemîn, Ligel Kurdan mêvan û ezimandina mêvanan gelekî girîng e. Îro derfet zêde bûne ji ber vê yekî jî dema ku li mêvandariyê derbas dibe hinekî kêm bûye. Kesên xerîb kêm caran li malan dibin mêvan û bi şev li wir dimînin. Berê ne wisa bû, car hebû bi roj û mehan mêvan li malê dihatin ezimandin. Wekî kevneşopiyekê odeya mêvanan hebû -hîn jî li gelek deveran heye- û mêvan bi mazûbanê xwe re li wir dima. Jin, nediçûn aliyê mêran. wekî weynên zayenda civakî, berpirsiyariyên amadekirin û rakirina şîvê, firaq û xizmetên din ên mêvanan (wekî şûştina kincên wan, rastkirina nivînên wan; hewandina hespên wan û hwd.) re mijûl dibûn. Lê belê dema mêvan bi dilxweşî ji wê malê vediqetiyan hemû gotinên xweş û dia ji bo mêrê malê dikirin.

Li gorî dabeşkirina weynên zayenda civakî divê keç li diya xwe, kur li bavê xwe herin û wekî wan bijîn. Wekî,

"Aş digere bi avê, keçik dike karê dê, lawik dike yê bavê." "95

"Keçik teşiya dê radike, lawik tîra bavê radike." "96

"Dayikê çing e, keçikê wing e." ${ }^{\prime 97}$ Keçik di şopa diya xwe de diçin.

85 - Oncu, Gotinên Pêşiyan, 373.

86 - Oncu, Gotinên Pêşiyan de Jin, 37.

87 - Oncu, Gotinên Pêşiyan de Jin, 36.

88 - Abdurrahman Aydoğdu, Gotinên Pêşiyan, (Stenbol: Weşanên na, 2014), 93.

89 - Occo Mahabad, "Gotinên Pêşiyan”, (31 Çile 2021).

90 - J4, Hevpeyvîna rû bi rû, 25 Çile 2021.

91 - J5, Hevpeyvîna rû bi rû, 25 Çile 2021.

92 - Pîrê Kal, "Gotinên Bijare”, Kovara War, (30 Çile 2021).

93 - Reşî̂, "Kürdlerde Durub-i Emsal", 304.

94 - Oncu, Gotinên Pêşyiyan de Jin, 164.

95 - Oncu, Gotinên Pêşiyan, 23.

96 - Mazî, Ferhenga Gotinên Pêşiyan, 252.

97 - Oncu, Gotinên Pêşiyan, 133. 
“Keçik teşiya dê dirêsê, lawik tîr û kevanê bavê xwe radike.” Di vê gotina pêşiyan de ligel dabeşkirina weynan, dabeşkirina pişseyan jî xûya dike. Teşîrêstin, karê nav malê ye û xistine stûyê keçikan. Herweha nêçirvanî jî karê derve ye û xistine stûyê mêran.

Ew yek carinan bi xizmên din ve jî tê girêdan. Wekî,

"Keç li metan, law li xalan tên." 98

"Keçik yê xelkê ne" "Wate: Keçik heta ku zewicîne wekî emanet in li mala bavê xwe. Lê ji bo kuran ne weha ye. Dibêjin, "Mala zêra xerab dibit, mala kura xerab nabit". ${ }^{100}$ Keçik, heta ku zewicîne di bin baskê dê û bavê de ne. Piştî ku dergîstî bûn an jî zewicîn civak wan dixe bin berpirsiyariya dergîstî, xwesû û xwezûran. Ew yek guherîna weyniyên zayenda civakî jî bi xwe re tîne. Di gotinên pêşiyan de ew rewş weha rawe dibe:

"Keçika di mala bavê xwe de mîna pezê emanet e."

"Keçika bêbav, çiyayê bêav”, "Keçika bêdê wek çiyayê bêrê., Ew herdu gotinên pêşiyan bawerî û nêrîna civakê a derbarê keçikan de nîşan didin. Li gorî van gotinan, keçika ku bêyî dê û bavê xwe mezin bibe, ji edet, kevneşopî û cerebeyên jiyanê bêpar radibe. Bêkêr e.

“Keçika bidergîstî di gund de, mîna gayê av di bin de. "102 Li gorî vê gotinê, piştî ku keçik dergîstî bû êdî nikare azad tevbigere. Neçar e li gorî daxwaziyên dergîstiyê xwe tevbigere.

Di çarçoveya gotinên pêşiyan de em dikarin bibêjin ku di hemû weynên zayenda civakî de jinên baş û nebaş hene. Civak li gorî çand, kevneşopî, dem, cih, rewşa nirxên siyasî û civakî, statû û berjewendiyên xwe jinan wekî jinên baş û nebaş tasnîf dike. Wekî li jor jî hate behskirin, hemû bi weynên zayenda civakî ve girêdayî ne. Carinan heman jin li gel hinekan dibe "jina baş", li gel hin kesên din jî dibe "jina nebaş." Li gorî gotinên pêşiyan jina baş ew e ku li gorî weynên xwe yên zayenda civakî rabe û ji bo kes û civaka xwe bibe xwedî pesn û paye. Lewra berpirsiyarên wê yên nav malê dikarin malê xera bikin an jî ava bikin. Serbilindî û rûreşiya mêran bi tevgera jina (hevsera) wan ve girêdayî ye. Di vê mijarê de gelek gotinên pêşiyan hene. Wekî,

“Jin, meran dike wezîr jî̀; dike rezil jî.,"103

“Jin dikarin mêran rakin jî, daxin jî." ${ }_{104}$

"Jin dikare peyayê xwe rezîl jî bike, ezîz jî bike." "105

"Jin çavlider e, mêr xwelîser e."

"Jin ku le mal tine win, mal nawe mal.",107

"Jin heye malkir e, jin heye malxwur e." 108

Bi alîkariya hin gotinên pêşiyan em dikarin derbarê civakê, pêwendiya jin û mêran û rewşa nav malê ji nêzik ve nas bikin. Ji ber ku her gotineke pêşiyan serborî û çîrokeke wê heye. ${ }^{109}$ Li gorî weynên zayenda civakî jinên nebaş hin taybetiyên wan hene:

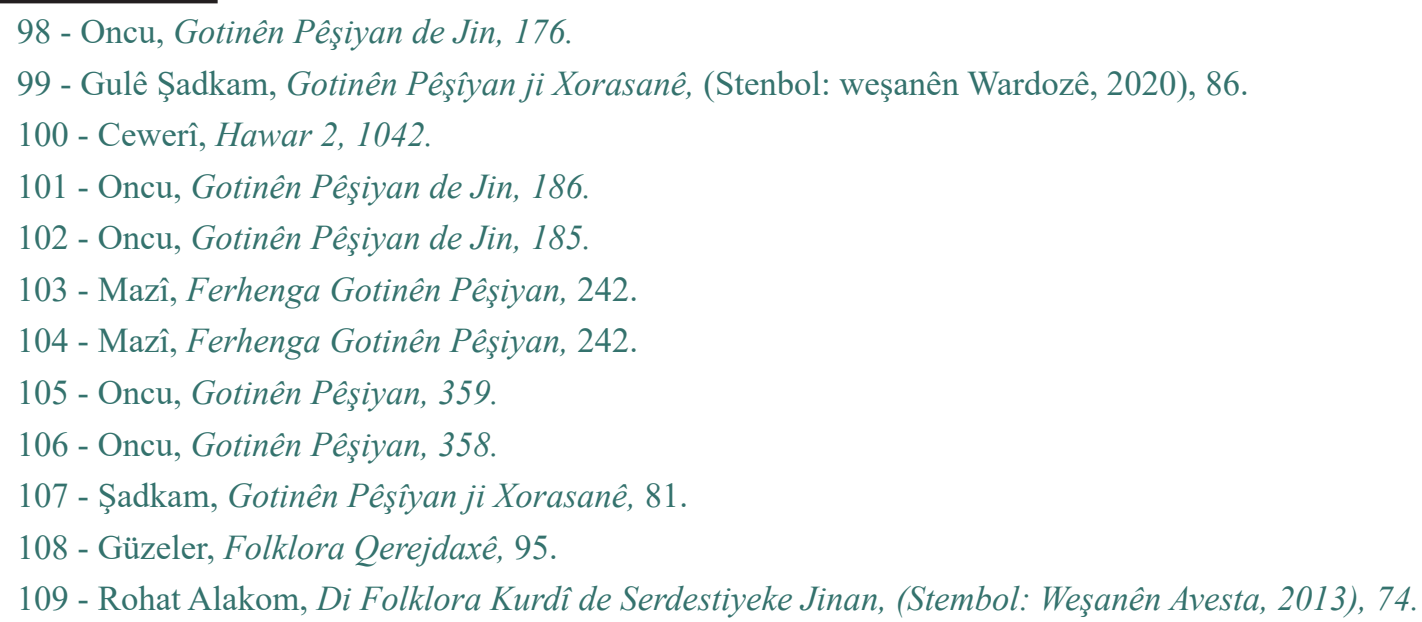


Jina nebaş li pêşiya mêrê xwe dibe asteng. Wekî,

"Şeytanên yetîman heft in, ên jinan heftê û heft in. " 110 Wate: Jin, zêde dipeyivin. Ev yek jî dibe sedema gelek pirsgirêkan.

“Jina xerab qeyd e, ne tê kuştin ne tê berdan." ${ }_{111}$ Wate: Dema ku mêr ji jineke xerab re bizewice nikare bi hêsanî ji wê pak bibe.

"Jina xerab udiya ${ }^{112}$ canê mêrê xwe ye." 113

Jina nebaş tiral (zexel) e. Wekî,

"Gavan ber bi mal bû, jina gavên bi kar bû. " ${ }^{114}$ Wate: Jin heta êvarê ji xwe re raza ye û li kêfa xwe nihêrtiyê. Lê dema ku hatina mêrê wê nêzik dibe, radibe dest bi kar dike ji bo ku xwe jêhatî binimîne.

Jin hene ku nikarin kar û barê xwe safî bikin. Wekî,

“Jin hene wek mirîşka kor in." "115 Wate: Heta êvarê dilebitin lê nikarin karê xwe sererast bikin.)

"Jin dirêse, belê nizane hember yek bike." "116 Wate: Jin çiqas bixebite jî bi ser nakeve, mihtacê mêr e.) Ev gotina pêşiyan û gelekên din jinê "qels" û wekî hevcedarê "mêran" nîşan didin.

Jin zû têne xapandin. Wekî,

"Kesê jinek bîne, divê an tûrek perê wî an jî barek derewê wî hebe." ${ }_{117}$ Wate: Jin bi pereyan ango derewan zû dikarin werin xapandin.

Başî û nebaşiya jinan bi kêrhatina wan a li gorî daxwazên mêr û civakê diyar dibe. Jina baş kêfa mêrê xwe tîne lê jina nebaş jiyanê li mêrê xwe diherimîne. Jina baş ew e kul i malê be, bi kar û barên malê ve mijûl bibe, di çarçoveya ku mêrê wê û civakê diyarkirî de tevbigere û di biryargirtinê de ne xwedî gotin be ye. Wekî,

“Jina baş jîn e, jina nebaş jan e. " 118

"Jina baş bextiyar dike, ya xerab extiyar dike." "119

"Jina baş yar û war e, ya xerab jehra mar e"

Başî û nebaşiya mêr ya di malê de di dest jinê de ye. Wekî,

"Jina baş, mêrê nebaş baş dike." "121

"Avaya malan li ser kevaniya malan."

"Jin hîmê malê ye." 123

Jinên bî/jinebî (ku mêrê wan mirine), jinên revandî û jinên berdayî (ku ji mêrê xwe qetiyane) di çavê civakê de piranî jinên "nebaş" in. Wekî,

110 - Oncu, Gotinên Pêşiyan de Jin, 245.

111 - Oncu, Di gotinên Pêşiyan de Jin, 159.

112 - udî: Heyberê xwînxwar ê efsûnî. Bi gumaneke mezin ji peyva tirkî ya "öcü” hatiye û ketiye nav devoka Semsûrê. Di jiyana rojane de ji bo kesên xerab ku mirov nikare pêsîra xwe jê veke jî tê gotin.

113 - Oncu, Di gotinên Pêşiyan de Jin, 159.

114 - Güzeler, Folklora Qerejdaxê, 67.

115 - Oncu, Gotinên Pêşiyan, 318.

116 - Mazî, Ferhenga Gotinên Pêşiyan, 241.

117 - Occo Mahabad, “Gotinên Pêşiyan”, (31 Çile 2021).

118 - Oncu, Gotinên Pêsiyan, 359.

119 - Kal, "Gotinên Bijare”. http://kovarabir.com/pire-kal-gotinen-bijare/ 15.05.2021.

120 - Kal, "Gotinên Bijare". http://kovarabir.com/pire-kal-gotinen-bijare/ 15.05.2021.

121 - Oncu, Di gotinên Pêşiyan de Jin, 136.

122 - Oncu, Di gotinên Pêşiyan de Jin, 14.

123 - Oncu, Di gotinên Pêşiyan de Jin, 119. 
“Jina bî, wekî girara bêxwê ye." "124

"Jina revandî, mîna rûnê helandî; jina bi dawet $\hat{u}$ def, mîna rûnê bi kef."

“Jina berdayî kera bindorê ye." 126 Ango jina berdayî naye bîra kesî. Nirxa wê kêm tê dîtin.

Arîşeya weynên zayenda civakî, ne tenê di nav mêr û jinan de herweha di nava jinan de jî heye. Gelek caran, di nava bûk û xwesûyê de niqaş derdikevin. Li gorî xwesûyan piştî ku bûk tê ser malê kurên wan guh nadin diya xwe. Wekî,

"Dayê buhik ${ }^{127}$ e, jinê ruhik e." 128

"Mala pirjin, xerabû ji bin.",129

"Jin derkete ser serî, dê avête ber derî"”|30 Wate: Piştî ku mêr zewicîn, nirxa diya wî kêm dibe. Lewre, bêtir nirxê dide jina xwe.

"Dergûş li dayikan ne bar e." "131 Wate: Dergûş ango anîna zarokan ji bo dayikan cihê kêfxweşiyê ye.

Xanima malê, çiqas baş be jî ji hewiya xwe hez nake. Lewra dibe şîrikê hevjîna wê. Loma dibêjin,

"Hewî gulokek rîs be, li ser pişta kewarê be, dîsa jî qehr e. "'32

Helbet hin gotinên pêşiyan ku wekheviya jin û meran derdixin pêş jî hene. Wekî, "Jin û mêr, tevr û bêr”'133 û "Şêr şêr e, çi jin e çi mêr e." ${ }^{134}$ ku behsa wekheviya jin û mêran dikin. Lê hejmara wan kêm e û mijara xebateke din in.

124 - Oncu, Di gotinên Pêşiyan de Jin, 142.

125 - Güzeler, Folklora Qerejdaxê, 96.

126 - Mazî, Ferhenga Gotinên Pêşiyan, 243.

127 - Buhik: Heybera efsûnî ku mirovan dixwe. Ji ber vê yekê mirov jê ditirsin û dixwazin dûrî wê bikevin. Bi taybetî ji bo ku zarokan ji hinek tişt û tevgeran dûr bixin, dibêjin "Buhik hate te".

128 - Oncu, Di gotinên Pêşiyan de Jin, 52.

129 - Oncu, Gotinên Pêşiyan, 466.

130 - Mazî, Ferhenga Gotinên Pêşiyan, 242.

131 - Oncu, Gotinên Pêşiyan, 147.

132 - Oncu, Gotinên Pêşiyan, 318.

133 - Halil Karataş, Kürt Atasözlerinde Kadın Kavramı, (Ankara: RUSEN Rusya Araştırmaları Enstitüsü, 2016$), 7$.

134 - Şadkam, Gotinên Pêşîyan ji Xorasanê, 137. 


\section{ENCAM}

Gotinên pêşiyan, encama çand û serhatiyên gel in. Di nav zimanê hemû gelan de gotinên bi vî rengî hene û hişmendiya civakê destnîşan dikin. Gelek caran, di heman mijarê de gotinên pêşiyan ên dijwate hatine gotin. Gelê Kurd jî di encama çand û serhatiyên hezarsalan de bi deh hezaran gotinên pêşiyan gotine û bi riya çanda devkî gihandine heta roja îro.

Her gotinek pêşiyan tecrûbeyekê -di heman demê de- nêrîn û hişmendiyekê rawe dike. Di vê çarçoveyê de weynên zayenda civakî jî di nav gotinên pêşiyan ên kurdî de cihê xwe girtine. Em dikarin bibêjin ku gelek gotinên pêşiyan li ser weynên jinan yên zayenda civakî hatiye rawestiyane.

Jin, ji kesayetekê zêdetir wekî endameke malbatê derdikeve pêş. Jin gelek caran wekî berpirsiyarê karê nav malê û gihandina zarokan hatiye pêşkêşkirin.

Di nav malê de wekî keçik, jin, dayik, xwesî, dapîr bi weynên zayenda civakî radibe û di heman demê de li hemberî mêrê xwe, merivên xwe û civakê berpirsiyarê "namûsê" ye. Di vê çarçoveyê de, jina ku bi weynên xwe yên zayenda civakî rabe "jina baş" e. Jina ku li dijî weynên ku ji bo wê hatine terxankirin rabe jî "jina nebaş “e.

Nêrîna li ser zarokên keçik di çarçoveya zewacê de ye. Keçik, her gav wekî namzeteke jinbûnê ango dayiktiyê hatine dîtin. Jin, ji zaroktiyê heta navsaliyê bi hişmendiya zewacê têne gihandin. Ezebmayîna keçikan wekî qisûr û kêmaniyeke mezin tê pejirandin û her gav dibe mijara rexneyê.

Di avakirina malbatê de kesayet herî girîng esaleta jinê ye. Lewra li gorî hişmendiya pederşahiye, " $Y a$ ku merivan ezîz jî dike, rezîl jî dike jin e." 135

Ji bo têgihiştina wate û nirxa gotinên pêşiyan divê her yek ji wanan di çarçoveya bûyer, rewşa çandî, şaristaniya wê serdemê, dem û devera ku lê hatiye gotin de were şîrovekirin. Gotinên pêşiyan, neynika civakê ne. Ji bo fêmkirina rabirdûya civakê girîng in lê ew naye wê wateyê ku hemû rast in ango xelet in. Divê berî ku em wan bi kar bînin di parzûnê hiş, aqil û zanistiyê re derbas bikin. 


\section{JÊDER}

Alakom, Rohat. Di Folklora Kurdî de, Serdestiyeke Jinan. Stenbol: Weşanên Avesta, 2013.

Amos, Dan Ben. "Şartlar ve Çevre İçinde Folklorun Bir Tanımına Doğru”. çev. Metin Ekici. Halk Biliminde Kuramlar ve Yaklaşımlar 1. ed. M. Öcal Oğuz, Metin Ekici, Nebi Özdemir, Gülin Öğüt Eker, Selcan Gürçayır. 37-57 Ankara: Geleneksel Yayınları, 2006.

Assmann, Jan. Kültürel Bellek, çev. Ayşe Tekin, İstanbul: Ayrıntı Yayınları, 2001.

Aydoğdu, Abdurrahman. Gotinên Pêşiyan. Stenbol: Weşanên na, 2014. CambridgeDictionary, https://dictionary.cambridge.org/tr/s\%C3\%B6z$1 \% \mathrm{C} 3 \% \mathrm{BCk} /$ ingilizce/an-apple-a-day-keeps-the-doctor-away 02.05.2021.

Borak, Mustafa. Ferhenga Biwêjan, Stenbol: Weşanên Enstitûya Stenbolê. 2005.

Çifçi, Sema-Bayram Değer, Vasfiye- Bayram, Nurcan. "Üniversite Öğrencilerinin Toplumsal Cinsiyet Algıları: Mardin Artuklu Üniversitesi Örneği”. Uluslararası Hakemli Ekonomi Yönetimi Araştırmaları Dergisi. 14 (2017), 37-60.

Çifçi, Tekin. "Kültür, Sanat ve Siyaset Adamı Olarak Celadet Ali Bedirhan”. Kürdoloji Akademik Çalışmalar, ed. Hasan Karacan. Cilt 2/482522. Ankara: Yarg1 Yayınevi, 2016.

Çifçi, Tekin. Baweriyên Gelêrî û Dermanê Kurmancî Li Herêma Semsûrê. Diyarbekir: Zanîngeha Dîcleyê, Enstitûya Zanistên Civakî, Teza Doktorayê, 2019.

Dêrşewî, Mele Mehmûd. Miştaxa Çiya Ji Gotinên Pêşiya, wer. Serbest Zaxoyî. Stockholm: 1989.

Dorson, Richard M. Thoms, W.J. "William John Thoms ve "Folklor” Başlıklı Yazısı”. Çev. Serpil Aygün Cengiz, Halk Biliminde Kuramlar ve Yaklaşımlar. ed. M. Öcal Oğuz, Selcan Gürçayır. 11-16. Ankara: Geleneksel Yayınları, 2003.

“Gotinên Pêşiyan”, https://www.bernamegeh.org/2019/08/02/kurtce-atasozleri-gotinen-pesiyan/ 12.08.2021.

Granbom-Herranen Liisa. "Women`s Place in Finnish Proverbs from Childhood”. Folklore: Electronic Journal of Folklore 46 (2010), 95-110. Güncel Türkçe Sözlük, https://sozluk.gov.tr/

Güzeler, Mehmet. Folklora Qerejdaxê, İstanbul: Weşanên Nûbihar, 2021.

Hin Gotinên Pêşiyan, https://candname.com/gotinen-pesiyan-3/ 12.06.2021.

Hirsch, E. D, Kett, Joseph F, Trefil, James. "Proverb", The New Dictionary of Cultural Literacy. Boston, New York: Houghton Mifflin Company, 2002.

Kal, Pîrê. “Gotinên Bijare”, Kovara War. 30 Çile 202. http://kovarabir.com/pire-kal-gotinen-bijare/

Karacan, Hasan, Ayus İhsan. “Kürtçe ve Türkçe Atasözlerinin Karşılaştırmalı Bir Biçimde İncelenmesi”. Kürdoloji Akademik Çalışmalar. ed. Hasan Karacan Cilt 1/113-158. Diyarbakır: Dicle Üniversitesi Basımevi, 2016.

Karacan, Hasan-Recep Erdinç, “Rus ve Kürt Atasözlerinin Kelime ve Anlam Karşılaştırması”, Dicle Üniversitesi İlahiyat Fakültesi Dergisi 16/2 (2014), 539-583.

Karataş, Halil. Kürt Atasözlerinde Kadın Kavramı, Ankara: RUSEN Rusya Araştırmaları Enstitüsü Yayınları, 2016.

Keskin, Necat. Folklor û Edebiyata Gelêrî. Stembol: Weşanên Avesta, 2009.

Lomotey, Benedicta Adokarley-Chachu, Sewoenam. "Gender ideologies and power relations in proverbs: A cross-cultural study", Journal of Pragmatics 168 (2020), 69-80.

Mazî, Çiya. Ferhenga Gotinên Pêşiyan. Stenbol: Weşanên Elma, Sibat 2005.

Mieder, Wolfgang. Proverbs Speak Louder Than Words: Wisdom in Art, Culture, Folklore, History, Literature and Mass Media. New York: Peter Lang Press, 2008.

Mihyedîn, Mela. "Di Gotinên Pêşiyan de Jin -1”, https://candname.com/di-gotinen-pesiyan-de-jin-1/ 12.07.2021.

Ojo, Arewa, E. Alan, Dundes. "Proverbs and the Etnograpy of Speaking Folklore". American Anthropologist, 66/6 (1964), 70-85.

Oncu, Mehmet. Di Gotinên Pêşiyan de Jin, Van: Weşanên Sîtav, 2020.

Oncu, Mehmet. Gotinên Pêşiyan. Stenbol: Weşanên Enstitûya Stenbolê, 2009.

Oxford Learners Dictionaries, "Proverb”. Erişim 29 Ocak 2021. https://www.oxfordlearnersdictionaries.com/definition/english/prover$\mathrm{b} ? \mathrm{q}=$ proverb

Öztürk, Mustafa. "Vekolînek Derbarê Gotinên Peşiyan Ên Kurmancî De”, Mukaddime 6/2 (2015), 319-337.

Parlaktuna, İnci. “Türkiye’de Cinsiyete Dayalı Mesleki Ayrımcılığın Analizi”, Ege Akademik Bakış Dergisi 10/4 (Ekim 2010), $1217-1230$.

Powell, Gary N.-Greenhaus, Jeffrey H. "Sex, gender, and decisions at the family-work interface”. Journal of management 36/4 (2010), 1011-1039.

Reşîd, Law. "Kürdlerde Durub-i Emsal”. Jîn, Kovara Kurdî-Tirkî, Kürtçe- Türkçe Dergi, ed. Mehmed Emin Bozarslan. Uppsala: Weşanxana Deng, 1985.

Sanauddin, Noor. School of Social and Political Sciences. Glasgow: University of Glasgow, The College Social Sciences, PhD, 2015. http:// theses.gla.ac.uk/6243/ 
Sarwet, Rasul. "Gender and Power Relationships in the Language of Proverbs: Image of a Woman". FWU Journal of Social Sciences. Vol.9, No.2. 52. (Winter 2015), 52-62.

Şadkam, Gulê. Gotinên Pêşîyan ji Xorasanê, Stenbol: Weşanên Wardozê, 2020.

Tawûzparêz (C. Elî Bedirxan), "Proverbes”. Hawar. ed. Firat Cewerî Cild 2/1042. Stockholm: Weşanên Nûdem, 1998.

Yıldırım, Kadri hwd. Ji Destpêkê Heta Niha Folklora Kurdî. Stenbol: Weşanên Enstitûya Zimanên Zindî ya Zanîngeha Mardin Artuklu, 2013. 


\section{EXTENDED ABSTRACT}

Proverbs are defined as short, meaningful, stereotyped and artistic words that reflect the experience, prejudices and superstitions of the society. It is possible to come across similar stereotypes in all societies and cultures around the world. There are thousands of proverbs on different subjects in Kurdish culture as well. These proverbs also reflect the economic, cultural, social and psychological situation of the Kurds. Gender perception and gender roles direct the functioning of daily life. Business and professional life is shared between women and men according to gender roles and continues traditionally. Proverbs, from birth to death on everyone, every status (such as wife and husband, mother, stepmother, widow, aunt, uncle, niece, grandfather, elder, host, slave, etc.), of nature and characteristics people (such as jealousy, kindness, cowardice, dishonesty, theft, equality, hatred, oppression, brotherhood, etc.) have been mentioned.

In the past, some studies were carried out on the compilation, explanation or classification of Kurdish proverbs. For example, Mela Mehmûdê Bayezîdî wrote a book in the middle of the 19th century called "Kilam, Berîte, Gotinên Pêşiyan". Law Rashid and Hîlmiyê Siwêregî in the years 1918 and 1919 scientifically focused on the proverbs of the Kurds and made an attempt to divide them. Law Reşîd, in Jîn magazine, has divided these proverbs according to topics such as social, heroic, literary, judicial, domestic, agricultural, weather, historical, legendary, canonical and narrative proverb and with their Turkish stopped at their meaning. Celadet Elî Bedirxan, published in Hawar magazine in Damascus and translated the proverbs of the Kurds, their meanings and interpretations into French.

This study focuses on the image of good women and bad women in Kurdish proverbs in the context of gender. For this purpose, thousands of verbal and written Kurdish proverbs from different sources were reviewed, and proverbs dealing with gender-based roles of women and men were determined.

Business and professional life is shared between women and men according to gender roles and continues traditionally. As in all areas of life, social gender roles appear in Kurdish proverbs. A masculine language prevails throughout Kurdish proverbs and the image of good women and bad women is shaped within the framework of the patriarchal approach. There are some proverbs that humiliate the woman's personality. For example, "Jinikê got mela; mehra min mehkem nebire, ez dest-pê nesekinî me." (The woman said to the imam: Don't make my marriage strong, because my right and left are not clear. I am not a decent person.), "Dersê digire ji jinê, dihere dibe dijminê." (He takes ideas from women, goes and becomes hostile to everyone.) or "Mêrê xeberjin, berxê ber bizin, şûva bi kizin tu tişt jê dernayê." Meaning: Husbands who act according to what their wife says cannot be successful. Because women do not know the right way, only men can decide on the spot.) On the other hand, there are proverbs that point to the importance of women in the family. For example, "Jin hîmê malê ye." (Woman is the foundation of the home.) or "Jin ku le mal tine win, mal nawê mal." (If there is no woman in the house, it is not a house).

Women, in the home as daughters, husbands, mothers, sisters-in-law, grandparents with social gender images and at the same time are responsible for "honor" towards their husbands, husbands and society. For example," Jina baş yar û war e, ya xerab jehra mar e." (The good woman is a lover and a country, a bad one is snake venom) or "Ya ku merivan ezîz jî dike, rezîl jî dike jin e." (What makes a man saint and disgraceful is a woman.)

In this context, the woman who rises to the top with her gender imagery is a "good woman". A woman who rises up against the images that have been set for her is also a "bad woman". 
As a result, women who behave in accordance with the gender roles that society and especially men assign to women have the image of "good woman". Women who oppose the gender roles assigned to them and act according to their individual will, feelings and thoughts have the image of "bad woman".

There are also proverbs that focus on gender equality. For example, "Şêr şêr $e$, çi jin $e$ çi mêr $e$." (A lion is a lion, male or female). Meaning: Men and women are equal in all respects.

However, such proverbs are few and are the subject of another study. 